\title{
Fatores que interferem na adesão ao tratamento do Diabetes Mellitus tipo 2
}

\author{
Factors that interfer with adhesion to the treatment of Diabetes Mellitus type 2 \\ Factores que interfieren con la adhesión al tratamiento de la Diabetes Mellitus tipo 2
}

Recebido: 17/12/2021 | Revisado: 24/12/2021 | Aceito: 29/12/2021 | Publicado: 07/01/2022

\author{
Patrícia Tavares dos Santos \\ ORCID: https://orcid.org/0000-0002-5431-0683 \\ Universidade Federal de Lavras, Brasil \\ E-mail: patttavares@hotmail.com \\ Rafaela Côrrea Pereira \\ ORCID: https://orcid.org/0000-0001-8795-6109 \\ Universidade Federal de Lavras, Brasil \\ E-mail: rafacpereira@gmail.com \\ Priscila Missaki Nakamura \\ ORCID: https://orcid.org/0000-0003-4993-4317 \\ Instituto Federal de Educação, Ciências e Tecnologias, Brasil \\ E-mail: missaki.naka@gmail.com \\ Rodrigo Ferreira de Moura \\ ORCID: https://orcid.org/0000-0002-6617-6434 \\ Universidade Federal de Lavras, Brasil \\ E-mail: rodrigo.moura@dsa.ufla.br
}

\begin{abstract}
Resumo
Objetivo: Este estudo buscou identificar e definir quais os fatores que interferem na adesão às atividades de autocuidado com o Diabetes Mellitus tipo 2 (DM2). Métodos: Participaram deste estudo 103 adultos, ambos os sexos, com diagnóstico de DM2, que foram avaliados através de questionário socioeconômico, Questionário de Atividades de Autocuidado com o Diabetes (QAD) e Questionário Internacional de Atividade Física (IPAQ). O estudo foi realizado no município de Três Corações-MG, no período de agosto de 2018 a outubro de 2019. Resultados: No questionário QAD a atividade com maior adesão foi relacionada à ingestão de medicamentos $(6,52$ dias $\pm 1,52)$ e a menor adesão foi a atividade relacionada à prática de exercícios físicos específicos $(1,29$ dias $\pm 2,11)$. O questionário IPAQ determinou que 66,0\% dos participantes atingiram as recomendações de atividade física da Associação Americana de Diabetes. Identificando os fatores que interferem na adesão às atividades de autocuidado com DM2 e à recomendação de atividade física, foram encontrados através da análise de cluster, que o baixo grau de escolaridade $(\mathrm{p}<0,001)$ e baixos índices de hemoglobina glicada $(\mathrm{p}=0,032)$ foram significativos na discriminação dos agrupamentos quando se considerou as variáveis de não adesão às atividades de autocuidado. O grau de escolaridade $(\mathrm{p}<0,001)$, por sua vez, foi fator significativo na discriminação, considerando as variáveis de não adesão às recomendações de atividade física. Conclusão: Foi possível demostrar que fatores sociais e clínicos podem influenciar na adesão ao tratamento dos pacientes diagnosticados com DM2.
\end{abstract}

Palavras-chave: Diabetes Mellitus Tipo 2; Autocuidado; Atividade física.

\begin{abstract}
Objective: This study aimed to identify and define which factors interfere with adherence to type 2 Diabetes Mellitus (DM2) self-care activities. Methods: A hundred three (103) adults both sexes, diagnosed with Type 2 Diabetes Mellitus, have participated in this study. They were assessed through a socioeconomic questionnaire, Diabetes SelfCare Activities Questionnaire (DSCAQ) and International Physical Activity Questionnaire. (IPAQ). The study was carried out in the city of Três Corações-MG, from August 2018 to October 2019. Results: In the DSCA questionnaire, the activity with the highest adherence was related to medication intake $(6,52$ days $\pm 1,52)$ and the lowest adherence was the activity related to the practice of specific physical exercises $(1,29$ days $\pm 2,11)$. The IPAQ questionnaire determined that $66.0 \%$ of the participants reached the physical activity recommendations from the American Diabetes Association. By identifying the factors that interfere with adherence to self-care activities with DM2 and physical activity recommendation it was found through the cluster analysis Pearson's chi-square that the education low level ( $\mathrm{p}$ $<0,001)$ and glycated hemoglobin low index $(\mathrm{p}=0,032)$ were significant in the discrimination of groups when considering the variables of non-adherence to self-care activities. The education low level $(\mathrm{p}<0,001)$, in turn, was a significant factor in discrimination of groups, considering the variables of non-adherence to physical activity recommendations. Conclusion: It was possible to demonstrate that social and clinical factors may influence adherence to the patients diagnosed with DM2 treatment.
\end{abstract}

Keywords: Diabetes Mellitus, Type 2; Self-care; Physical activity. 


\section{Resumen}

Objetivo: Este estudio buscó identificar y definir qué factores interfieren con la adherencia a las actividades de autocuidado en la Diabetes Mellitus tipo 2 (DM2). Métodos: Participaron 103 adultos, de ambos sexos, diagnosticados de DM2, evaluados mediante un cuestionario socioeconómico, un Cuestionario de Actividades de Autocuidado en Diabetes (QAD) y un Cuestionario Internacional de Actividad Física (IPAQ). El estudio se llevó a cabo en la ciudad de Três Corações-MG, de agosto de 2018 a octubre de 2019. Resultados: En el cuestionario QAD, la actividad con mayor adherencia se relacionó con la ingesta de medicamentos $(6,52$ días $\pm 1,52)$ y la menor adherencia fue la actividad relacionada con la práctica de ejercicios físicos específicos $(1,29$ días $\pm 2,11)$. El cuestionario IPAQ determinó que el 66,0\% de los participantes cumplían con las recomendaciones de actividad física de la Asociación Estadounidense de Diabetes. Identificando los factores que interfieren con la adherencia a las actividades de autocuidado con DM2 y la recomendación de actividad física, el análisis de conglomerados mostró que la baja escolaridad ( $p<0,001)$ y los bajos niveles de hemoglobina glucosilada $(p=0,032)$ fueron significativos en la discriminación de grupos al considerar las variables de no adherencia a las actividades de autocuidado. El nivel de educación ( $p<0,001$ ), a su vez, fue un factor significativo en la discriminación, considerando las variables de no adherencia a las recomendaciones de actividad física. Conclusión: se pudo demostrar que factores sociales y clínicos pueden influir en la adherencia al tratamiento de los pacientes diagnosticados de DM2.

Palabras clave: Diabetes Mellitus tipo 2; Autocuidado; Actividad física.

\section{Introdução}

O Diabetes Mellitus (DM) é uma séria ameaça à saúde mundial. Os números de pacientes diagnosticados com essa doença multiplicam anualmente, chegando a 463 milhões em 2019, com previsões alarmantes para os próximos anos. Porém, com diagnóstico precoce e cuidados apropriados, as alterações metabólicas do diabetes podem ser administradas e suas complicações atrasadas ou evitadas (World Health Organization, 2016; International Diabetes Federation, 2019; American Diabetes Association, 2019).

O Diabetes Mellitus tipo 2 (DM2) é o mais frequente e corresponde a 90 - 95\% dos casos. É causado por uma disfunção nas células $\beta$ do pâncreas associada à resistência dos tecidos periféricos à insulina, posteriormente ocorre a redução das células $\beta$ do pâncreas reduzindo a produção deste hormônio (World Health Organization, 2019; Sociedade Brasileira de Diabetes, 2019).

A partir do diagnóstico do DM2, é necessário a realização de atividades de autocuidado ao longo da vida para prevenir ou atrasar as complicações da doença e melhorar a qualidade de vida. Essas atividades englobam dieta saudável, atividade física, uso correto de medicamentos, monitoramento da glicemia e cuidado com os pés (Lu et al., 2016; Borba et al., 2018; World Health Organization, 2003; Simpson \& Eurich, 2016).

Para o controle da glicemia e a redução das complicações é essencial a adesão do indivíduo ao tratamento (Michels et al., 2010). Diversos fatores podem influenciar em uma melhor ou pior adesão às atividades de autocuidado com o diabetes, como fatores psicológicos, físicos e sociais (Marinho et al., 2018).

Gibicoski et al. (2020) observa que a falta de adesão às práticas de autocuidado pode acarretar em maiores índices de internações hospitalares e surgimento de agravos da doença. De outro modo, a prática adequada destas atividades acelera o resultado terapêutico e melhora o controle do metabolismo.

Por isso, conhecer o nível de adesão às atividades de autocuidado e os fatores que influenciam esta adesão é importante para intervir e melhorar a eficácia do tratamento do DM2.

\section{Metodologia}

\subsection{Desenho do estudo}

Trata-se de um estudo transversal descritivo, com abordagem quantitativa (Pereira et al, 2018), realizado na cidade de Três Coraç̃̃es - MG, no sudeste do Brasil localizado a 300km de Belo Horizonte, capital do estado de Minas Gerais. A cidade possui população total estimada em 78.913 pessoas e um Índice de Desenvolvimento Humano de 0,744 (Instituto Brasileiro de 
Geografia e Estatística, 2020).

\subsection{Aspectos éticos}

Foi aprovado pelo Comitê de Ética em Pesquisa com Seres Humanos da Universidade Federal de Lavras com parecer $\mathrm{n}^{\mathrm{o}}$ 2.779.200, de acordo com a resolução 466/2012 CNS/MS. Os pacientes foram informados sobre o estudo e os que concordaram em participar assinaram o Termo de Consentimento Livre e Esclarecido.

\subsection{Coleta de dados}

O presente estudo foi realizado no período de agosto de 2018 a outubro de 2019, abordando adultos, maiores de 18 anos, de ambos os sexos, com diagnóstico de DM2. A coleta dos dados foi realizada de forma individual, em local reservado, através da aplicação dos questionários e consulta aos prontuários.

As entrevistas foram realizadas na Associação dos Diabéticos de Três Corações, uma associação filantrópica criada em 1993, que em parceria com a Prefeitura Municipal de Três Corações e voluntários atendem os pacientes diagnosticados com DM2 no município. Possui profissionais de diversas especialidades como endocrinologia, clínica geral, nutrição, fisioterapia, psicologia, enfermagem, podologia e terapia ocupacional. São realizados cerca de 150 procedimentos semanais. Os pacientes que frequentam a Associação recebem o atendimento médico, pré-consulta com técnica de enfermagem para aferição da pressão arterial e glicemia capilar, palestras semanais com nutricionistas, reuniões mensais com a direção da Associação para palestras, oficinas terapêuticas e organização das normas.

Os 103 participantes foram submetidos a entrevistas individuais por meio dos questionários: sociodemográfico e clínico, Questionário de atividades de autocuidado com o Diabetes (QAD), Questionário Internacional de Atividade Física (IPAQ) versão curta. Os dados coletados em prontuários foram medicamentos prescritos e exames bioquímicos como hemoglobina glicada (HbA1c) realizado a menos de três meses da data da entrevista.

\subsection{Questionários}

\subsubsection{Socioeconômico e clínico}

Foi aplicado um questionário socioeconômico e clínico contendo informações como idade, escolaridade, renda mensal familiar, estado civil, medicamentos prescritos, tipo de aquisição dos medicamentos prescritos, complicações diagnosticadas e HbA1c.

\subsubsection{Adesão ao autocuidado do Diabetes Mellitus}

O Questionário de Atividades de Autocuidado com o Diabetes (QAD) foi aplicado para mensurar as atividades de autocuidado preconizadas pela American Diabetes Association (ADA) aos pacientes entrevistados. O QAD é um questionário traduzido e adaptado do Summary of Diabetes Self-care Activities Questionnaire (SDSCA), possui seis dimensões e 15 itens de avaliação do autocuidado com o diabetes. Os entrevistados relataram com que frequência eles realizaram as atividades nos últimos sete dias. As respostas variaram de 0 a 7 , sendo zero a situação menos desejável e sete a mais favorável. Nos itens que questionam sobre o consumo de alimentos ricos em gorduras e doces, os valores foram invertidos, por exemplo, se a ingestão foi de sete dias, o valor corresponde a zero dias de adesão, se foram seis dias de ingestão, o valor corresponde a um dia de adesão e assim sucessivamente (Michels et al., 2010).

Marinho et al. (2018) consideram adesão à prática das atividades de autocuidado com Diabetes, a adesão a todos os itens específicos do QAD por cinco dias ou mais na semana. 


\subsubsection{Atividade física}

Para avaliar a realização de atividade física ou sedentarismo foi utilizado o International Physical Activity Questionnaire (IPAQ) - versão curta. Esse questionário avalia o tempo que os entrevistados realizam atividades físicas vigorosas, moderadas e caminhadas, avalia também o tempo gasto sentado no trabalho, em casa, na escola ou no tempo livre. O resultado de cada pergunta será multiplicado pelos dias da semana e somados (Matsudo et al., 2001). Assim, será verificado se o tempo gasto com a realização de atividades físicas por semana atende a recomendação da Associação Americana de Diabetes de 150 minutos ou mais de atividade física moderada a vigorosa por semana, ao longo de pelo menos três dias na semana (American Diabetes Association, 2019).

\subsection{Análise de dados}

Os dados foram analisados por técnicas estatísticas diferentes: para descrever os dados sociodemográficos e clínicos foram utilizadas média, frequência e desvio-padrão; para testar a capacidade de predizer quais os fatores interferem na adesão às atividades de autocuidado no tratamento do Diabetes Mellitus tipo 2 foram utilizadas regressão logística binária e análise de cluster. Essas análises foram realizadas no software Statistical Package for Social Sciences - SPSS. O nível de significância adotado foi $\mathrm{p}<0,05$.

$\mathrm{Na}$ análise hierárquica de agrupamentos realizou-se um procedimento para identificar os clusters hierárquicos 2 a 3 clusters, através do método de Ward. A solução de 3 clusters foi selecionada por demonstrar a melhor correlação entre as variáveis. Testes qui-quadrado comprovaram as diferenças significantes entre os 3 grupos.

A divisão da amostra em 3 clusters ficou discriminada da seguinte forma, clusters 1 e 2 compostos por indivíduos que não aderiram às atividades de autocuidado do DM2 e cluster 3 composto por indivíduos que aderiram às atividades de autocuidado do DM2.

O agrupamento em clusters é uma técnica usada para atribuir elementos em grupos, assim os elementos que são agrupados dentro de um mesmo cluster são muito parecidos entre si. O objetivo é encontrar pontos semelhantes e diferentes no conjunto de dados (Van de Velden et al., 2017).

\section{Resultados}

\subsection{Características dos participantes}

A amostra foi composta de 103 adultos com diagnóstico de DM2. As características estão descritas na Tabela 1. A média de idade foi de 61,01 anos ( $\pm 10,09)$. A maioria dos participantes eram do sexo feminino, $61,2 \%$ (n=63), casados $(67 \%$, $\mathrm{n}=69$ ), moradores da zona urbana do município de Três Corações $(96,1 \%, n=99)$, com grau de escolaridade fundamental incompleto $(57,3 \%, \mathrm{n}=59)$ e com renda familiar de 1 a 1,5 salários-mínimos $(50,5 \%, \mathrm{n}=52)$.

O medicamento mais utilizado para o controle glicêmico foi da classe das biguanidas (40,3\%, $\mathrm{n}=77$ ), seguida pelas sulfonilureias $(27,2 \%, \mathrm{n}=52)$. A maioria dos participantes adquiriram seus medicamentos pelo Sistema Único de Saúde $(53,4 \%, \mathrm{n}=55)$. O tempo médio de diagnóstico de DM2 foi de 11,4 anos $( \pm 8,75)$. E a maioria não apresentou complicações relacionadas ao DM2 $(62,2 \%, \mathrm{n}=69)$. Dos participantes que relataram complicações, a mais comum é a neuropatia diabética $(18,9 \%, \mathrm{n}=21)$. A média de nível sérico de HbA1c foi de 8,33\% ( $\pm 1,93)$. 
Tabela 1 - Caracterização dos indivíduos com DM2 conforme dados clínicos e sociodemográficos. Três Corações - MG, Brasil, $2021(\mathrm{n}=103)$.

\begin{tabular}{|c|c|c|}
\hline Características & Frequência ou Média & Percentual ou Desvio Padrão \\
\hline \multicolumn{3}{|l|}{ Sexo } \\
\hline Feminino & 63 & $61,2 \%$ \\
\hline Masculino & 40 & $38,8 \%$ \\
\hline Idade & 61,01 & $\pm 10,09$ \\
\hline \multicolumn{3}{|l|}{ Estado Civil } \\
\hline Casados & 69 & $67 \%$ \\
\hline Solteiros & 6 & $5,8 \%$ \\
\hline Divorciados & 8 & $7,8 \%$ \\
\hline Viúvos & 20 & $19,4 \%$ \\
\hline \multicolumn{3}{|l|}{ Escolaridade } \\
\hline Analfabeto & 5 & $4,9 \%$ \\
\hline Fundamental incompleto & 59 & $57,3 \%$ \\
\hline Fundamental completo & 18 & $17,5 \%$ \\
\hline Médio completo & 12 & $11,7 \%$ \\
\hline Superior & 9 & $8,7 \%$ \\
\hline \multicolumn{3}{|l|}{ Renda familiar } \\
\hline 1 - 1,5 salários mínimos & 52 & $50,5 \%$ \\
\hline 1,5 - 3 salários mínimos & 33 & $32 \%$ \\
\hline Mais que 3 salários mínimos & 18 & $17,5 \%$ \\
\hline \multicolumn{3}{|l|}{ Endereço } \\
\hline Zona urbana & 99 & $96,1 \%$ \\
\hline Zona rural & 4 & $3,9 \%$ \\
\hline Tempo de diagnóstico DM & 11,4 anos & $\pm 8,75$ \\
\hline $\mathrm{HbA1c}$ & 8,33 & $\pm 1,93$ \\
\hline \multicolumn{3}{|l|}{ Medicamentos } \\
\hline Insulina & 42 & $22 \%$ \\
\hline Sulfonilureias & 52 & $27,2 \%$ \\
\hline Inibidores DPP4 & 13 & $6,8 \%$ \\
\hline Biguanidas & 77 & $40,3 \%$ \\
\hline Inibidores de SGLT2 & 5 & $2,6 \%$ \\
\hline Tiazolidinedionas & 1 & $0,5 \%$ \\
\hline Associações & 1 & $0,5 \%$ \\
\hline \multicolumn{3}{|l|}{ Complicações DM } \\
\hline Nenhuma & 69 & $62,2 \%$ \\
\hline Amputação MMII & 1 & $0,9 \%$ \\
\hline Doença cardiovascular & 7 & $6,3 \%$ \\
\hline Nefropatia & 4 & $3,6 \%$ \\
\hline Neuropatia & 21 & $18,9 \%$ \\
\hline Pé diabético & 1 & $0,9 \%$ \\
\hline Retinopatia & 8 & $7,2 \%$ \\
\hline
\end{tabular}

Fonte: Elaborado pelos autores (2021).

\subsection{Questionário de Atividades de Autocuidado com o Diabetes - QAD}

O Questionário de Atividades de Autocuidado com o Diabetes avalia seis dimensões do autocuidado e a Tabela 2 apresenta a média de dias de adesão aos itens específicos. O item que teve maior adesão foi relacionado à ingestão de medicamentos com média de 6,52 dias e o item com menor adesão foi relacionado à prática de exercício físico específico como nadar, caminhar, andar de bicicleta, sem incluir suas atividades em casa ou em seu trabalho, com média de 1,29 dias.

Utilizando o critério de Marinho et al. (2018) a adesão por 5 dias ou mais na semana em todos os itens foi praticada por $11,7 \%(\mathrm{n}=12)$ dos participantes. 
Tabela 2 - Adesão às atividades de autocuidado ao Diabetes. Três Corações - MG, Brasil, 2021 (n=103)

\begin{tabular}{|c|c|}
\hline Atividades de autocuidado & Média do $\mathrm{n}^{\circ}$ de dias \\
\hline \multicolumn{2}{|l|}{ Alimentação geral } \\
\hline Seguir uma alimentação saudável & $3,53( \pm 2,96)$ \\
\hline Seguir uma orientação nutricional & $3,44( \pm 2,89)$ \\
\hline \multicolumn{2}{|l|}{ Alimentação específica } \\
\hline Consumir cinco ou mais porções de frutas e/ou vegetais & $2,15( \pm 2,93)$ \\
\hline Consumir alimentos ricos em gorduras & $5,12( \pm 2,34)$ \\
\hline Consumir doces & $5,45( \pm 1,95)$ \\
\hline \multicolumn{2}{|l|}{ Atividade física } \\
\hline Prática de atividade física durante pelo menos 30 minutos & $2,18( \pm 2,30)$ \\
\hline Prática de exercício físico especifico & $1,29( \pm 2,11)$ \\
\hline \multicolumn{2}{|l|}{ Monitorização da glicemia } \\
\hline Aferição da glicemia capilar & $2,40( \pm 2,70)$ \\
\hline Aferição da glicemia capilar o $\mathrm{n}^{\circ}$ de vezes recomendado no dia & $1,99( \pm 2,85)$ \\
\hline \multicolumn{2}{|l|}{ Cuidado com os pés } \\
\hline Exame dos pés & $5,88( \pm 2,26)$ \\
\hline Exame dos sapatos antes de calçá-los & $5,54( \pm 2,79)$ \\
\hline Secar entre os dedos dos pés depois de lavá-los & $6,32( \pm 2,01)$ \\
\hline \multicolumn{2}{|l|}{ Medicação } \\
\hline Ingestão de todos os medicamentos do diabetes, conforme recomendado & $6,52( \pm 1,52)$ \\
\hline Tabagismo & Sim \\
\hline Fuma & $0(87 \%) \quad 04801$ \\
\hline
\end{tabular}

Fonte: Elaborado pelos autores (2021).

\subsection{Questionário Internacional de Atividade Física - IPAQ}

O Questionário Internacional de Atividade Física foi utilizado para determinar o nível de atividade física dos participantes. A Tabela 3 descreve os resultados das análises do questionário IPAQ. A média de tempo gasto com a prática de atividades vigorosas foi de 57,5 minutos por semana, com atividades moderadas foi de 410,1 minutos por semana e caminhadas foi de 183,8 minutos por semana. Dos 103 participantes, 66\% (n=68) atenderam a recomendação da Associação Americana de Diabetes para a realização de atividade física por 150 minutos por semana (American Diabetes Association, 2019).

Tabela 3 - Adesão à atividade física segundo questionário IPAQ. Três Corações - MG, Brasil, 2021 (n=103)

\begin{tabular}{ll}
\hline Atividades & Tempo médio (minutos) \\
\hline Vigorosas & 57,5 \\
Moderadas & 410,1 \\
Caminhadas & 183,8 \\
\hline Recomendação de Atividade Física & Percentual (N) \\
\hline Atende & $66 \%(68)$ \\
Não atende & $34 \%(35)$ \\
\hline
\end{tabular}

Fonte: Elaborado pelos autores (2021).

\subsection{Variáveis associadas à adesão ou não adesão}

A análise de cluster possibilitou a discriminação da amostra em três clusters, sendo que os participantes que aderiram às atividades de autocuidado do DM2 se agruparam no cluster 3 e os que não aderiram às atividades foram agrupados nos clusters 1 e 2 , conforme Tabelas 4 
Research, Society and Development, v. 11, n. 1, e29711124861, 2022

(CC BY 4.0) | ISSN 2525-3409 | DOI: http://dx.doi.org/10.33448/rsd-v11i1.24861

Tabela 4 - Fatores associados à adesão às atividades de autocuidado segundo análise de cluster. Três Corações - MG, Brasil, $2021(n=103)$.

\begin{tabular}{|c|c|c|c|c|}
\hline Variável & Cluster 1 & Cluster 2 & Cluster 3 & $\mathrm{P}$ valor \\
\hline Faixa etária & & & & 0,096 \\
\hline $31-40$ anos & $3(3,4 \%)$ & $0(0 \%)$ & $0(0 \%)$ & \\
\hline $41-50$ anos & $13(14,6 \%)$ & $0(0 \%)$ & $1(8,3 \%)$ & \\
\hline $51-60$ anos & $27(30,3 \%)$ & $0(0 \%)$ & $4(33,3 \%)$ & \\
\hline $61-70$ anos & $31(34,8 \%)$ & $0(0 \%)$ & $7(58,3 \%)$ & \\
\hline $71-80$ anos & $13(14,6 \%)$ & $2(100 \%)$ & $0(0 \%)$ & \\
\hline $81-90$ anos & $2(2,2 \%)$ & $0(0 \%)$ & $0(0 \%)$ & \\
\hline Sexo & & & & 0,923 \\
\hline Feminino & $55(61,8 \%)$ & $1(50 \%)$ & $7(58,3 \%)$ & \\
\hline Masculino & $34(38,2 \%)$ & $1(50 \%)$ & $5(41,7 \%)$ & \\
\hline Estado Civil & & & & 0,112 \\
\hline Solteiro & $4(4,5 \%)$ & $0(0 \%)$ & $2(16,7 \%)$ & \\
\hline Casado & $62(69,7 \%)$ & $1(50 \%)$ & $6(50 \%)$ & \\
\hline Divorciado & $5(5,6 \%)$ & $1(50 \%)$ & $2(16,7 \%)$ & \\
\hline Viúvo & $18(20,2 \%)$ & $0(0 \%)$ & $2(16,7 \%)$ & \\
\hline Endereço & & & & 0,721 \\
\hline Zona urbana & $85(95,5 \%)$ & $2(100 \%)$ & $12(100 \%)$ & \\
\hline Zona rural & $4(4,5 \%)$ & $0(0 \%)$ & $0(0 \%)$ & \\
\hline Renda familiar & & & & 0,617 \\
\hline 1 a 1,5 salários & $44(49,4 \%)$ & $2(100 \%)$ & $6(50 \%)$ & \\
\hline 1,5 a 3 salários & $30(33,7 \%)$ & $0(0 \%)$ & $3(25 \%)$ & \\
\hline Mais que 3 salários & $15(16,9 \%)$ & $0(0 \%)$ & $3(25 \%)$ & \\
\hline Escolaridade & & & & $0,001 *$ \\
\hline Analfabeto & $3(3,4 \%)$ & $2(100 \%)$ & $0(0 \%)$ & \\
\hline Fundamental incompleto & $55(61,8 \%)$ & $0(0 \%)$ & $4(33,3 \%)$ & \\
\hline Fundamental completo & $15(16,9 \%)$ & $0(0 \%)$ & $3(25 \%)$ & \\
\hline Ensino médio completo & $10(11,2 \%)$ & $0(0 \%)$ & $2(16,7 \%)$ & \\
\hline Ensino Superior completo & $6(6,7 \%)$ & $0(0 \%)$ & $3(25 \%)$ & \\
\hline Uso de insulina & & & & 0,962 \\
\hline Sim & $36(40,4 \%)$ & $1(50 \%)$ & $5(41,7 \%)$ & \\
\hline Não & $53(59,6 \%)$ & $1(50 \%)$ & $7(58,3 \%)$ & \\
\hline Uso de metformina & & & & 0,079 \\
\hline Sim & $64(71,9 \%)$ & $1(50 \%)$ & $12(100 \%)$ & \\
\hline Não & $25(28,1 \%)$ & $1(50 \%)$ & $0(0 \%)$ & \\
\hline $\mathrm{N}^{\mathrm{o}}$ de medicamentos & & & & 0,644 \\
\hline Até 6 medicamentos & $65(73 \%)$ & $1(50 \%)$ & $10(83,3 \%)$ & \\
\hline 7 a 10 medicamentos & $20(22,5 \%)$ & $1(50 \%)$ & $1(8,3 \%)$ & \\
\hline Mais de 10 medicamentos & $4(4,5 \%)$ & $0(0 \%)$ & $1(8,3 \%)$ & \\
\hline $\begin{array}{l}\text { Tipo de aquisição de } \\
\text { medicamentos }\end{array}$ & & & & 0,298 \\
\hline SUS & $48(53,9 \%)$ & $0(0 \%)$ & $7(58,3 \%)$ & \\
\hline Particular & $41(46,1 \%)$ & $2(100 \%)$ & $5(41,7 \%)$ & \\
\hline Tempo de diagnóstico & & & & 0,421 \\
\hline Menos de 10 anos & $49(55,1 \%)$ & $2(100 \%)$ & $9(75 \%)$ & \\
\hline 11 a 20 anos & $29(32,6 \%)$ & $0(0 \%)$ & $3(25 \%)$ & \\
\hline Mais de 21 anos & $11(12,4 \%)$ & $0(0 \%)$ & $0(0 \%)$ & \\
\hline
\end{tabular}


Research, Society and Development, v. 11, n. 1, e29711124861, 2022

(CC BY 4.0) | ISSN 2525-3409 | DOI: http://dx.doi.org/10.33448/rsd-v11i1.24861

\begin{tabular}{ccccc}
\hline Complicação & & & 0,497 \\
Sim & $29(32,6 \%)$ & $0(0 \%)$ & $5(41,7 \%)$ & \\
Não & $60(67,4 \%)$ & $2(100 \%)$ & $7(58,3 \%)$ & \\
\hline HbA1c & & & & $0,032^{*}$ \\
Até 6,5\% & $13(14,6 \%)$ & $0(0 \%)$ & $3(25 \%)$ & \\
$6,5-10 \%$ & $62(69,7 \%)$ & $0(0 \%)$ & $7(58,3 \%)$ & \\
Mais que 10\% & $14(15,7 \%)$ & $2(100 \%)$ & $2(16,7 \%)$ & \\
\hline
\end{tabular}

Fonte: Elaborado pelos autores (2021).

As variáveis significativas em relação a variável dependente Adesão às atividades de autocuidado foram Escolaridade $(\mathrm{p}<0,001), \mathrm{HbA1c}(\mathrm{p}=0,032)$. Assim os participantes com menor grau de escolaridade e menor faixa de HbA1c foram agrupados no cluster 1 de não aderentes.

Quanto à adesão às recomendações de atividade física, segundo a análise de cluster, foi possível discriminar a amostra em três clusters, sendo que os participantes que aderiram às recomendações de atividade física foram agrupados nos cluster 1 e 2 e os que não aderiram às recomendações foram agrupados no cluster 3 , conforme Tabela 5.

Tabela 5 - Fatores associados à adesão à atividade física segundo análise de cluster. Três Corações - MG, Brasil, 2020 $(n=103)$.

\begin{tabular}{|c|c|c|c|c|}
\hline Variável & Cluster 1 & Cluster 2 & Cluster 3 & $\mathrm{P}$ valor \\
\hline Faixa etária & & & & 0,466 \\
\hline $31-40$ anos & $1(1,5 \%)$ & $0(0 \%)$ & $2(5,7 \%)$ & \\
\hline $41-50$ anos & $10(14,9 \%)$ & $0(0 \%)$ & $4(11,4 \%)$ & \\
\hline $51-60$ anos & $22(32,8 \%)$ & $0(0 \%)$ & $9(25,7 \%)$ & \\
\hline $61-70$ anos & $26(38,8 \%)$ & $0(0 \%)$ & $12(34,3 \%)$ & \\
\hline $71-80$ anos & $7(10,4 \%)$ & $1(100 \%)$ & $7(20 \%)$ & \\
\hline $81-90$ anos & $1(1,5 \%)$ & $0(0 \%)$ & $1(2,9 \%)$ & \\
\hline Sexo & & & & 0,436 \\
\hline Feminino & $42(62,7 \%)$ & $0(0 \%)$ & $21(60 \%)$ & \\
\hline Masculino & $25(37,3 \%)$ & $1(100 \%)$ & $14(40 \%)$ & \\
\hline Estado Civil & & & & 0,924 \\
\hline Solteiro & $4(6 \%)$ & $0(0 \%)$ & $2(5,7 \%)$ & \\
\hline Casado & $46(68,7 \%)$ & $1(100 \%)$ & $22(62,9 \%)$ & \\
\hline Divorciado & $6(9 \%)$ & $0(0 \%)$ & $2(5,7 \%)$ & \\
\hline Viúvo & $11(16,4 \%)$ & $0(0 \%)$ & $9(25,7 \%)$ & \\
\hline Endereço & & & & 0,327 \\
\hline Zona urbana & $63(94 \%)$ & $1(100 \%)$ & $35(100 \%)$ & \\
\hline Zona rural & $4(6 \%)$ & $0(0 \%)$ & $0(0 \%)$ & \\
\hline Renda familiar & & & & 0,476 \\
\hline 1 a 1,5 salários & $32(47,8 \%)$ & $1(100 \%)$ & $19(54,3 \%)$ & \\
\hline 1,5 a 3 salários & $25(37,3 \%)$ & $0(0 \%)$ & $8(22,9 \%)$ & \\
\hline Mais que 3 salários & $10(14,9 \%)$ & $0(0 \%)$ & $8(22,9 \%)$ & \\
\hline Escolaridade & & & & $0,001^{*}$ \\
\hline Analfabeto & $3(4,5 \%)$ & $1(100 \%)$ & $1(2,9 \%)$ & \\
\hline Fundamental incompleto & $38(56,7 \%)$ & $0(0 \%)$ & $21(60 \%)$ & \\
\hline Fundamental completo & $16(23,9 \%)$ & $0(0 \%)$ & $2(5,7 \%)$ & \\
\hline Ensino médio completo & $4(6,0 \%)$ & $0(0 \%)$ & $8(22,9 \%)$ & \\
\hline Ensino Superior completo & $6(9,0 \%)$ & $0(0 \%)$ & $3(8,6 \%)$ & \\
\hline
\end{tabular}


Research, Society and Development, v. 11, n. 1, e29711124861, 2022

(CC BY 4.0) | ISSN 2525-3409 | DOI: http://dx.doi.org/10.33448/rsd-v11i1.24861

\begin{tabular}{|c|c|c|c|c|}
\hline Uso de insulina & & & & 0,480 \\
\hline Sim & $27(40,3 \%)$ & $1(100 \%)$ & $14(40 \%)$ & \\
\hline Não & $40(59,7 \%)$ & $0(0 \%)$ & $21(60 \%)$ & \\
\hline Uso de metformina & & & & 0,058 \\
\hline Sim & $54(70,1 \%)$ & $0(0 \%)$ & $23(29,9 \%)$ & \\
\hline Não & $13(50 \%)$ & $1(3,8 \%)$ & $12(46,2 \%)$ & \\
\hline $\mathrm{N}^{\circ}$ de medicamentos & & & & 0,974 \\
\hline Até 6 medicamentos & $49(73,1 \%)$ & $1(100 \%)$ & $26(74,3 \%)$ & \\
\hline 7 a 10 medicamentos & $15(22,4 \%)$ & $0(0 \%)$ & $7(20 \%)$ & \\
\hline Mais de 10 medicamentos & $3(4,5 \%)$ & $0(0 \%)$ & $2(5,7 \%)$ & \\
\hline $\begin{array}{l}\text { Tipo de aquisição de } \\
\text { medicamentos }\end{array}$ & & & & 0,560 \\
\hline SUS & $36(53,7 \%)$ & $0(0 \%)$ & $19(54,3 \%)$ & \\
\hline Particular & $31(46,3 \%)$ & $1(100 \%)$ & $16(45,7 \%)$ & \\
\hline Tempo de diagnóstico & & & & 0,762 \\
\hline Menos de 10 anos & $41(61,2 \%)$ & $1(100 \%)$ & $18(51,4 \%)$ & \\
\hline 11 a 20 anos & $20(29,9 \%)$ & $0(0 \%)$ & $12(34,3 \%)$ & \\
\hline Mais de 21 anos & $6(9 \%)$ & $0(0 \%)$ & $5(14,3 \%)$ & \\
\hline Complicação & & & & 0,771 \\
\hline Sim & $22(32,8 \%)$ & $0(0 \%)$ & $12(34,3 \%)$ & \\
\hline Não & $45(67,2 \%)$ & $1(100 \%)$ & $23(65,7 \%)$ & \\
\hline HbA1c & & & & 0,197 \\
\hline Até $6,5 \%$ & $11(16,4 \%)$ & $0(0 \%)$ & $5(14,3 \%)$ & \\
\hline $6,5-10 \%$ & $43(64,2 \%)$ & $0(0 \%)$ & $26(74,3 \%)$ & \\
\hline Mais que $10 \%$ & $13(19,4 \%)$ & $1(100 \%)$ & $4(11,4 \%)$ & \\
\hline
\end{tabular}

Fonte: Elaborado pelos autores (2021).

Sobre a variável dependente Recomendação de Atividade Física somente a Escolaridade foi significativa ( $<00,001)$, assim os participantes com maior grau de escolaridade (Ensino médio completo) pertencem ao cluster 3, no qual foram mais prevalentes os participantes que não atenderam às recomendações de atividade física

\section{Discussão}

O objetivo principal do tratamento do diabetes mellitus é alcançar níveis glicêmicos adequados, evitando assim as complicações relacionadas à uma hiperglicemia constante. Para que o controle glicêmico seja alcançado são necessários cuidados e práticas que melhorem a qualidade de vida do indivíduo com diagnóstico de DM2 (Kassahun et al., 2016).

Pereira et al. (2021) reforça que implantar estratégias de promoção autocuidado como atividade física e plano alimentar saudável se faz importante devido aos altos índices de DM2 no Brasil.

Avaliando a adesão às atividades de autocuidado através do questionário $\mathrm{QAD}$, foi encontrado uma maior adesão às atividades relacionadas à ingestão de medicamentos (6,52 dias) e uma menor adesão nas atividades relacionadas à prática de exercício físico específico como nadar, caminhar, andar de bicicleta (1,29 dias).

Nesta pesquisa, assim como no estudo realizado por Janoo e Khan (2018)' a maior adesão às atividades relacionadas à ingestão de medicamentos pode ser relacionada à influência exercida pela prescrição médica e por estar associada a um bom controle glicêmico.

Um estudo brasileiro realizado em Ribeirão Preto também obteve como resultado uma baixa adesão à prática de exercícios físicos específicos, que foi justificado pela ocorrência de complicações como úlcera e/ou amputações nos membros 
inferiores e fatores psicossociais como cansaço, falta de tempo, desânimo entre outros (Gomides et al., 2013). Para Ausili et al. (2017) a adesão ao exercício físico no DM2 encontra barreiras em diversos outros fatores como físicos, psicológicos e sociais. No presente estudo, podemos encontrar outras justificativas na amostra para a não realização de exercícios físicos por 5 dias ou mais na semana, como a idade, a maior parte da amostra está acima dos 60 anos (53,3\%) e a baixa renda, 50,5\% apresentaram uma renda menor que 1,5 salário mínimo. Esses fatores podem dificultar a realização de exercícios físicos específicos como natação, musculação, andar de bicicleta, devido às comorbidades e às dificuldades para o custeio.

Por outro lado, verificamos que 66\% $(n=68)$ atenderam as recomendações da ADA para a realização de atividade física, que inclui qualquer movimento que gera gasto energético (American Diabetes Association, 2019). Resultado semelhante a um estudo australiano no qual 57\% dos participantes com DM2 atenderam a essas recomendações (Nolan et al., 2016). Encontramos também que o grau de escolaridade pode influenciar na adesão à atividade física. Segundo a análise de cluster os participantes com maior grau de escolaridade pertenceram ao agrupamento de não aderentes.

Assim como Gomides et al. (2013), encontramos que a escolaridade influencia a adesão às atividades de autocuidado. Foi verificado que os indivíduos com menores graus de escolaridade (analfabetismo e fundamental incompleto) pertenceram ao agrupamento de não aderentes. Acredita-se que a baixa escolaridade pode dificultar o acesso às informações, à interpretação das instruções e à compreensão da relevância das atividades de autocuidado.

Segundo Janoo e Khan (2018), não há garantias de que a adesão às atividades de autocuidado resulte em um bom controle glicêmico. De modo interessante, nossos resultados revelaram que o grupo com menores faixas de HbA1c (abaixo de 6,5\%) estão no agrupamento de não aderentes. Isso implica na limitação do uso da HbA1c como indicador de boa adesão ao autocuidado. Infelizmente nosso estudo não permite responder o que causa essa divergência, mas questões como sintomas mais leves e fatores psicológicos como negação talvez estejam relacionados.

\section{Conclusão}

Os resultados encontrados revelam uma baixa adesão geral às atividades de autocuidado. Verificou-se que a escolaridade é o principal indicador de baixa adesão aos autocuidados. Além disso, a Hb1Ac de forma isolada não é um bom indicador para inferência sobre o autocuidado. $\mathrm{O}$ delineamento de futuras intervenções terapêuticas deveriam considerar esses fatores na elaboração do tratamento.

Assim fica evidente a necessidade de estudos futuros afim de identificar os motivos que justificam a adesão ou não adesão às práticas de autocuidado nos pacientes com DM2, bem como estudos longitudinais com intervenções educativas considerando as características dos pacientes.

\section{Referências}

American Diabetes Association. (2019). Diabetes Care: Standards of Medical Care in Diabetes. Diabetes Care. Virgínia.

Ausili, D., Bulgheroni, M., Ballatore, P., Specchia, C., Ajdini, A., Bezze, S., Di Mauro, S., \& Genovese, S. (2017). Self-care, quality of life and clinical outcomes of type 2 diabetes patients: an observational cross-sectional study. Acta Diabetologica, 54 (11), 1001-1008.

Borba, A. K. O. T., Marques, A. P. O., Ramos, V. P., Leal, M. C. C., Arruda, I. K. G., \& Ramos, R. S. R. S. (2018). Fatores associados à adesão terapêutica em idosos diabéticos assistidos na atenção primária de saúde. Ciência e Saúde Coletiva, 23 (3), 953-961.

Gibicoski, F. S., Moreschi, C., Rodrigues, S. O., Silva, S. O., Tolfo, A. P. D., \& Busnelo, E. D. S. (2020). Adesão de atividades de autocuidado de usuários com diabetes atendidos na atenção primária. Research, Society and Development, 9 (5), e20952977.

Gomides, D. S., Villas-Boas, L. C. G., Coelho, A. C. M., \& Pace, A. E. (2013). Autocuidado das pessoas com diabetes mellitus que possuem complicações em membros inferiores. Acta Paulista de Enfermagem, 26 (3), 289-293.

Instituto Brasileiro De Geografia e Estatística. Índice de desenvolvimento humano. Instituto Brasileiro De Geografia e Estatística

International Diabetes Federation. IDF Diabetes Atlas: Ninth edition 2019. (2019). International Diabetes Federation. 
Research, Society and Development, v. 11, n. 1, e29711124861, 2022

(CC BY 4.0) | ISSN 2525-3409 | DOI: http://dx.doi.org/10.33448/rsd-v11i1.24861

Jannoo, Z., \& Khan, N. M. (2018). Summary of Diabetes Self-care Activities: A confirmatory factor analytic approach. Primary Care Diabetes, 12 (5), $425-$ 431.

Kassahun, T., Gesesew, H., Mwanri, L., \& Eshetie, T. (2016). Diabetes related knowledge, self-care behaviours and adherence to medications among diabetic patients in Southwest Ethiopia: a cross-sectional survey. BMC Endocrine Disorders, 16 (1), 28.

Lu, Y., XU, J., Zhao, W., \& Han, H. (2016). Measuring Self-Care in Persons With Type 2 Diabetes: A Systematic Review. Evaluation and the Health Professions, 39(2), 131-184.

Marinho, F. S., Moram, C. B. M., Rodrigues, P. C., Leite, N. C., Salles, G. F., \& Cardoso, C. R. L. (2018). Treatment Adherence and Its Associated Factors in Patients with Type 2 Diabetes: Results from the Rio de Janeiro Type 2 Diabetes Cohort Study. Journal of Diabetes Research, (8970196), 1-8.

Matsudo, S., Araújo, T., Matsudo, V., Andrade, D., Andrade, E., Oliveira, L. C., \& Braggion, G. (2001). Questionário Internacional de Atividade Física (IPAQ): estudo de validade e reprodutibilidade no Brasil. Revista Brasileira de Atividade Física e Saúde, 6 (2), 5-18.

Michels, M. J., Coral, M. H. C., Sakae, T. M., Damas, T. B., \& Furlanetto, L. M. (2010). Questionário de Atividades de Autocuidado com o Diabetes: tradução, adaptação e avaliação das propriedades psicométricas. Arquivos Brasileiros de Endocrinologia \& Metabologia, 54(7), 644-651.

Nolan R C, Raynor, A. J., Berry, N. M., \& May, E. J. (2016). Self-reported physical activity using the International Physical Activity Questionnaire (IPAQ) in Australian adults with type 2 Diabetes, with and without peripheral neuropathy. Canadian Diabetes Association, 40 (6), $576-579$.

Pereira, A. S., Shitsuka, D. M., Parreira, F. J., \& Shitsuka, R. (2018). Metodologia da pesquisa científica. UFSM.

Pereira, P. F., Santos, J. C., Cortez, D. N., Reis, I. A., \& Torres, H. C. (2021). Avaliação das estratégias de educação em grupo e intervenção telefônica para o diabetes tipo 2. Revista da Escola de Enfermagem da USP, 55, e03746.

Simpson, S. H., Lin, M., \& Eurich, D. T. (2016). Medication Adherence Affects Risk of New Diabetes Complications: A Cohort Study. Annals of Pharmacotherapy, 50 (9), 741-746.

Sociedade Brasileira De Diabetes. (2019). Diretrizes da Sociedade Brasileira de Diabetes 2019-2020. Editora Clannad.

Van De Velden, M., D’enza, A. I., \& Palumbo, F. (2017). Cluster Correspondence Analysis. Psycometrika, 82 (1), $158-185$.

World Health Organization. (2003). Adherence to long-term therapies: evidence for action. World Health Organization.

World Health Organization. (2016). Global report on diabetes. World Health Organization.

World Health Organization. (2019). Classification of diabetes mellitus. World Health Organization. 